\title{
Experiences of Health Care Workers Regarding Maintenance of Confidentiality Concerning Hearing Challenged People in Selected Public Health Facilities in eThekwini District, South Africa
}

\author{
Snenhlanhla Kelly Dlamini ${ }^{1} \&$ Maureen Nokuthula Sibiya ${ }^{1}$ \\ ${ }^{1}$ Faculty of Health Sciences, Durban University of Technology, Durban, South Africa \\ Correspondence: Snenhlanhla Kelly Dlamini, Faculty of Health Sciences Durban University of Technology, P.O. \\ Box 1334, Durban, 4000, South Africa. Tel: 27-71-147-3394. E-mail: dsnenhlanhla780@gmail.com
}

Received: February 13, 2020 Accepted: March 16, 2020 Online Published: April 14, 2020

doi:10.5539/gjhs.v12n6p63 URL: https://doi.org/10.5539/gjhs.v12n6p63

\begin{abstract}
Communication between a health care provider and a patient is a pre-requisite for any intervention to occur in the process of health care. Deaf patients depend on interpreters when seeking health care. Confidentiality is an issue since interpreters are involved in the Deaf community. Health care workers (HCWs) are tasked to ensure that the delivery of information to the patient is accurate and kept confidential. A descriptive exploratory qualitative design was used to explore the experiences of HCWs regarding the maintenance of confidentiality for hard of hearing patients. The study population comprised HCWs who worked with hearing challenged patients. Purposive sampling, which is a type of non-probability sampling, was utilized to select the study participants from the population. This research study was conducted at two hospitals in eThekwini District. Structured interviews, using an interview schedule, were used to collect data. The actual sample size was 10 participants. Data was analysed using Tesch's eight steps of thematic analysis. Six major themes emerged from the data analysis namely training of health care workers, communication preferences for hard of hearing patients, communication challenges, health care worker-patient relationship, involvement of family members as interpreters and measures to maintain confidentiality. Majority of participants experienced communication challenges with patients who are hard of hearing since only few HCWs are trained in sign language. The findings of this study will contribute to addressing issues related to the maintenance of confidentiality of hard of hearing patients. This study does not claim to exhaust the subject but to raise awareness of new investigations in other realities. Further research in the larger representative population of HCWs and hard of hearing patients is necessary to make the health care system more accessible and communication with HCWs more satisfying and effective.
\end{abstract}

Keywords: Confidentiality, hard of hearing, health care worker, sign language, interpretation, therapeutic relationship, deaf, deaf culture, disability

\section{Introduction and Background}

Patient confidentiality is a vital attribute of professional practice that safeguards patients' rights (Richardson, 2014). Health care workers (HCWs) have a code of ethics and practice guidelines that govern the management of confidential information. Most deaf patients depend on interpreters when seeking health care, as they experience communication barriers with HCWs. Confidentiality remains an issue especially since interpreters are typically already involved in the deaf community and might know the individual personally (Richardson, 2014).

Patient confidentiality was introduced in the International Code of Medical Ethics in 1948 to produce a modern medical code for use worldwide (Mc Clelland \& Thomas, 2002). It aims to support and maintain patient confidentiality and to provide clarification of a doctor's duty in this area. Patient confidentiality is not introduced in medical practice only, but also exists for various medical professionals and international ethical years to the Hippocratic Oath. Patient confidentiality is an ancient concept, which goes back to ancient Egypt and to the physician Imhotep, who used to make his students take an oath not to divulge any secrets of their patients. Later, the Greek physician Hippocrates established the Hippocratic Oath for Doctors, which serves as a guideline for the modern medical profession code of ethics codes worldwide, such as the Geneva Declaration (1949). Patient confidentiality is a very old concept from the early days of medicine, which can be traced back over 2000 (Mc Clelland \& Thomas, 2002). 
Patient confidentiality is a form of informational privacy characterized by a special relationship such as the physician-patient relationship and the personal information obtained during this relationship should not be revealed to others without patient consent (Moore et al., 2007). Confidentiality relates to the concept that private and personal information must be kept secret and protected from any breach of security, which might occur. While one may be liable for invading a person's privacy, only those with information gained from the special confidential relationship have a duty to maintain its confidentiality, not share it without the patient's permission or in the absence of a compelling reason to do so. Confidentiality protects informational privacy interests by requiring receivers of information to restrict access to that information (Moore et al., 2007).

Patient confidentiality is a vital attribute of professional practice that safeguards patients' rights. It is deeply rooted in the codes of ethics and law and contributes to the duty of caring for a patient. The obligation to preserve and not reveal patient's information is both ethical and statutory for all. The South African Department of Health (2003), identifies four requirements to provide a patient with a confidential service. These entail protection of patient information; ensuring that only those who are directly involved in the patient's care gain access to their medical records; giving the patient adequate information about how their health cannot be disclosed to a third party; and lastly improvement of the first three requirements to achieve the best possible ways to protect, inform and provide choice by the patient (South Africa, Department of Health, 2003). Patient confidentiality also applies to computer and electronic records which are being utilized in some developed countries. Patient information is very sensitive, so the prevention of disclosure and the preservation of personal information leads to the development of trust and confidence between HCWs and patients, strengthening the relationship. A relationship based on trust maintains a patient's dignity and respects his/her faith and this encourages the patient to communicate openly and honestly with the HCWs about them. Health care professionals have a code of ethics and practice guidelines that govern the management of confidential information (O’Brien \& Charter, 2003).

HCWs are tasked to ensure that the delivery of information to the patient is accurate and that it is confidential. Confidentiality remains an issue especially since interpreters are involved in the deaf community and might know the individual personally (Richardson, 2014). The deaf community is a small community with defined familial, professional and social networks. Penn and de Andrade (2017) implies the need to explain and reinforce the need to protect the participants' rights to anonymity and confidentiality.

\section{Methods}

\subsection{Study Design}

Regarding the maintenance of confidentiality for hard of hearing patients. This research design offers a flexible yet structured approach in obtaining rich data about people's perspectives about any phenomena that are unexplored. It is widely used in social science research, education and health care. The qualitative research method enabled the researcher to be involved as an active participant of the process (Babbie \& Mouton, 2001). Grove et al. (2014), state that the aim is to give the participants a voice to interpret and represent their experiences in a coherent way that could be analysed by the researcher. The qualitative research method was selected as an effective way to inform related fields and to contribute to what is already known regarding the maintenance of confidentiality of hearing challenged patients.

\subsection{Study Setting}

This research study was conducted at two hospitals in eThekwini District. For confidentiality purposes, IALCH was coded as Hospital A and ADH as Hospital B. These hospitals were selected because the hard of hearing patients from various deaf societies and organisations are often referred to these hospitals as they have specialised ear, nose and throat (ENT) outpatient units as well as audiology departments. Hospital A is the only tertiary, central hospital in KwaZulu-Natal (KZN) with medical disciplines, surgical disciplines, mother and child health services as well as professions allied to medical support disciplines. Hospital B is one of the districts and regional hospitals in KZN, located at the south beach in Durban, which offers various health services.

\subsection{Population and Sampling}

Population is the entire group of people that is of interest to the researcher and that meet the criteria that the researcher is interested in studying. This research study targeted a population of HCWs at selected hospitals that provide services to hard of hearing patients. HCWs are all people engaged in actions whose primary intent is to enhance health. A HCW is a person who delivers care and service to the sick and ailing either directly as doctors and nurses or indirectly as aides, helpers, laboratory technicians or even medical waste handlers. At Hospital A, there are eight HCWs who provide services to hard of hearing patients in the ENT outpatient unit and at Hospital B, there are seven HCWs who provide services to hard of hearing patients in the ENT outpatient unit; thus, a total of 
15 HCWs. Ethical clearances were obtained prior to data collection. Pre-testing of the data collection tool was conducted including a few individuals who met the inclusion criteria but did not form part of the sample of the actual data collection. Purposive sampling was used in this research study. HCWs in ENT clinics and audiology departments were purposively selected as they can offer experiences about working with hard of hearing patients. HCWs, like doctors, were not available for participation since they were occupied with work. Due to the small population size, all consenting HCWs were included in the sample. However, data collection was guided by data saturation.

\subsection{Data Collection}

Permission was sought from the participants to use a portable voice recorder during the interview sessions. Interview schedules and an interview guide was prepared in consultation with the participants and interviews were conducted at a time that was convenient to the participants. Each session of the interview took 30 to 45 minutes.

\subsection{Data Analysis}

Field notes were done after the interview during data analysis. Data collected from the participants was analysed by using Tesch's eight steps of thematic analysis (Creswell, 2009). For data coding there were two coders, which was done by the researcher and was verified by the supervisor who is an expert in qualitative research.

\subsection{Trustworthiness}

Trustworthiness is the believability of the researcher's findings (Lincoln \& Guba, 2000). Credibility, transferability, dependability and confirmability are the key criteria for trustworthiness for a researcher to render a qualitative study credible. Credibility, the researcher had prolonged engagements with the participants to gain an adequate understanding of the phenomena being studied and to establish a relationship with the participants. Credibility was ensured through 'member checks' relating to the accuracy of the data on the spot of data collection at the end of data collection interviews (Lincoln \& Guba, 2000). Transferability was done through collection of a rich account of descriptive data which was provided such as the context in which the research was carried out, the setting, sample, sample size, type of sampling, inclusion and exclusion criteria, interview procedure and topics, changes in interview questions based on the research process and passages from the interview guide (Anney, 2014). Dependability was accomplished through carefully tracking the emerging research design and through keeping an audit trail that is detailed chronologically, of research activities and processes, and influences on the data collection and analysis. The audit trail was thereafter examined by the supervisor who is an expert in the field. Confirmability was ensured through audio recording all the interviews to ensure that the information provided by the participants were accurate and truthful. The researcher interpreted and analysed the data through identifying themes and sub-themes, which were supported using direct quotations from the interviews in order to eliminate subjectivity and bias.

\subsection{Ethical Considerations}

Ethical clearance (Reference number: IREC 098/19) was granted by the Durban University of Technology Research Ethics Committee. Gatekeeper permission approval was granted by the eThekwini Health District Office Health Research and Knowledge Management of KZN Department of Health and the Hospital Managers of the respective hospitals.

Each prospective participant was provided with a letter of information, which outlined the details of the study. The research aims and objectives as well as the role of the participants was explained to each participant and they were afforded an opportunity to ask questions and indicate their willingness to participate. Consent forms were signed by each participant prior to the interview. Confidentiality in the form of recordings and field notes was maintained by storage on the researcher's laptop with a code only known to the researcher.

\section{Results}

The participants consisted of 2 speech therapists, 1 audiologist, and 11 nurses from the two selected hospitals. All the participants were females. Their ages ranged from 25 to 49 years, and the length of service ranged from 6 months to 22 years.

Six major themes emerged from the data analysis namely, training of health care workers, communication preferences for hard of hearing patients, involvement of family members during consultation, communication challenges with hard of hearing patients, measures to maintain confidentiality and health care workers' relationship with hard of hearing patients. 


\subsection{Training of Health Care Workers}

Participants expressed diverse opinions regarding training on South African Sign Language (SASL). Most participants verbalised that they received minimal training on SASL, whilst the minority indicated that they had not received training. These participants indicated lack of training as one of the reasons for not being comfortable communicating with patients. Participants had the following to say:

"There's different variations of sign language, so we may not know all the variations of sign language, they may come from different places as well. That is a problem where they use different signs for different words as compared to us." (Participant 04, Hospital B, Chief Speech Therapists/Head of Department).

"We are comfortable with them because we have been trained how to assess and manage them, we did a small course at our university." (Participant 03, Hospital B, Audiologist).

"My interaction with them is pretty easy because I know sign language, and some may be pretty challenging especially if they not understanding written language. Usually the difficult cases, they usually come with somebody who can communicate with us verbally and even through written language to assist with interpretation. For me the issues come in when the patient now has limited vocabulary and when there's issues in terms of cognition that is huge challenge for us." (Participant 04, Hospital B, Chief Speech Therapist/HOD).

"So far I have never come across a case where it was just impossible to communicate or to get the necessary aim achieved. The big challenge would be the whole financial issue of having to go and come again for the second time if I could not get the message across." (Participant 03, Hospital B, Audiologist).

\subsection{Communication Preferences for Hard of Hearing Patients}

This theme emerged as the participants described the preferences of communication with various patients who also come with various language backgrounds when seeking health care. The findings revealed that HCWs experienced challenges when treating a hard of hearing patient who uses a different South African language, which the HCWs are not familiar with. The participants verbalised that it is also very challenging when they communicate with the patients who are foreigners who speak different African languages. They indicated how they check the patient's language preferences:

"When the patient comes, we greet the patient, if they are African, I use IsiZulu to greet and if it is not an African person I will greet in English, then I will see when they respond which language they prefer to use. It is then that I will start communicating in that language, but you find that sometime a patient will respond hesitantly only to find that they do not know the language you are greeting with then you ask if they are Sotho or Venda. If you know that language you start communicating with the patient but sometimes you find that a patient is deaf and cannot also understand the language that you are speaking which is a challenge." (Participant 09, Hospital A, Nurse).

"It is a challenge when patients communicate in African languages because we do not know how to communicate in such languages its better when they know a little bit of English." (Participant 06, Hospital A, Nurse).

"I have never found myself having a nurse to assist. There is always a family to assist if a patient cannot communicate in English." (Participant 02, Hospital B, Speech Therapist).

\subsection{Involvement of Family Members During Consultation}

This theme emerged as the participants mentioned various measures that they use in order to maintain confidentiality for hard of hearing patients during consultations. They expressed that the involvement of family members does assist the hard of hearing patient in terms of interpreting but prior to the consultation; the patient is asked if the family member should be involved. The participants reported that before the family member enters the consultation room, the patient is asked for permission since private and confidential information is going to be discussed. However, some hard of hearing patients require that some information like the HIV status not to be discussed when the family member is involved. This is noted in the following excerpts:

"....... when the patient cannot communicate in all forms cannot like read, write or understand whatever is being said, health care workers are forced to include the family member as an interpreter during consultation even if the patient can use sign language unfortunately no one in the department is trained on sign language." (Participant 05, Hospital A, Nurse).

"When the patient is called for their turn for consultation they usually come with the family member because the doctor will have to talk with the family member to interpret to the patient, so that the patient can understand everything that is being said by the doctor, it does not happen that the patient comes on their own to the hospital." (Participant 02, Hospital A, Nurse). 
"If they are attending with the family member and if they like the family member to be in the session we would allow it, they have to be comfortable with the family member. We ask them if they would like the family member to come as well." (Participant 03, Hospital B, Audiologist).

\subsection{Communication Challenges With Hard of Hearing Patients}

This theme emerged as the participants described strategies, they use to overcome communication challenges between hard of hearing patients. The sub-themes include the use of gestures and writing and the use of health care workers.

Participants reported that they used various gestures and writing to communicate with those hard of hearing patients who are literate. They also use non-verbal language such as eye contact and looking directly at the patients who can lip read. Participants used the following ways:

"..... the ones that use sign language, I use both sign language, gestures and lip reading, we have limited sign language but we use it like that, in cases where we really do not understand then we use the written mode of communication to communicate with them." (Participant 01, Hospital B, Speech Therapist).

"For patients who are hard of hearing we raise our voices and maintain eye contact so that even if they did not clearly hear what you were saying they can at least read your lips. For patients who are deaf, we also pay attention, maintain eye contact, read the patients facial expression to assess if what you are saying the patient can or cannot hear. We also try communicating through the little knowledge of sign language that we have tried to learn on our own." (Participant 12, Hospital A, Nurse).

"We try speaking to them if that fails then its pen and paper, we try to use writing to understand what they require, any problems they are having, why they came to the hospital, and if they have an appointment." (Participant 15, Hospital A, Nurse).

"First, verbally and then we try to use signs and gestures and if all of that fails then writing." (Participant 03, Hospital B, Audiologist).

\subsection{Measures to Maintain Confidentiality}

This theme emerged as the participants revealed the measures that they use to maintain confidentiality during consultation with hard of hearing patients. The results revealed that all consultations were conducted in a closed and soundproof room with only the HCWs involved in the patient's care present in the room. This is noted in the following excerpts:

"...closed rooms which are soundproof are used during consultation with the hard of hearing patients. Soundproof is important because sometimes we have to raise our voices for the patient to hear." (Participant 13, Hospital A, Nurse).

"We normally see them in a closed environment, we also conduct our testing's in a room away from everyone else and the feedback in the consultation room with the door closed." (Participant 01, Hospital B, Speech Therapist/Audiologist).

"The health care setting that we use admits one patient in a room per consultation which ensures that the other patients cannot hear what is being discussed about the patient during consultation." (Participant 06, Hospital A, Nurse).

\subsection{Health Care Workers' Relationship With Hard of Hearing Patients}

This theme emerged as the participants described their relationship with the hard of hearing patients. The sub-themes were the effect of an interpreter and the measures they use to ensure the health care worker-patient relationship especially when an interpreter such as a family member is involved. Participants expressed that it is a challenge to maintain their relationship with the hard of hearing patients when there is an interpreter involved as a third person in the consultation. They reported that it is time-consuming for the patient when the information is passed by the doctor to the nurse, to the interpreter and then to the hard of hearing patient. This is noted in the following excerpts:

"The interpreter also plays the role on the after-care part where they need to help the patient about the food, they need to eat for example ensure soft diet." (Participant 09, Hospital A, Nurse).

"A simple thing as a smile, greeting your patient and asking how they are feeling today. You form a bond with the patient and connect in a professional way; you respect and listen the patient." (Participant 06, Hospital B, Nurse).

“...when I take history of the patient, I may come across as empathetic and wanting to help the patient but I do not 
know whether my questions are conveyed in that manner, so with experience for me always the third person becomes an issue because I just feel there needs to be a relationship and the bond between the patient and the clinician." (Participant 04, Hospital B, Chief Speech Therapist/HOD).

"Having an interpreter who is close to the hard of hearing patient helps during the consultation but it increases the time the patient spends in the consultation room because the doctor will pass a message to the nurse, the nurse to the interpreter who will then pass it to the patient. You find that even five minutes is spent on one question." (Participant 08, Hospital A, Nurse).

\section{Discussion of Themes}

The findings of the current study revealed that minimal training of HCWs was identified in the health care setting. Simasiku and Nghitanwa (2017) argue that HCWs should be able to communicate effectively with deaf patients for effective quality nursing care. Only a few HCWs have been trained on communication with deaf and hard of hearing patients. They also state that effective communication between nurses and deaf patients is an essential element of nursing care yet nursing education on how to communicate with deaf patients is insufficient (Simasiku \& Nghitanwa, 2017). According to Machado et al. (2015), nurses must be prepared to communicate with deaf patients through sign language. Although communication is emphasized as one of the most important basic tools of this profession, courses in sign language have not been included in the undergraduate nursing courses (Machado et al., 2015).

Participants indicated that lack of sign language training makes it difficult to communicate with deaf patients. This finding is supported by the study in Rio de Janerio, which found that most nurses did not understand sign language because they were not trained in sign language (Machado et al., 2015). A study by Alselai and Alrashed (2015) found that HCWs were deficient in sign language due to lack of instructions in nursing schools, and training in the job on communication with hard of hearing patients. They further state that HCWs are insufficiently prepared to work with deaf patients whose primary mode of communication is sign language (Alselai \& Alrashed, 2015).

Sign language training for HCWs is the only way that can improve communication with deaf patients during the provision of health care. Through sign language communication the right information from the patients will be obtained and proper quality nursing care could be provided (Moore et al., 2007). The achievement of an inclusive health practice regarding deaf patients is dependent on the basic understanding of sign language and cultural and linguistic particularities of the deaf community, which are considered essential skills that must be taught during academic training of these professionals (Moore et al., 2007). The South African Department of Health (2005) highlights the importance of training all front line HCWs in sign language to improve communication. The theoretical concept used in this theme was the identification of a health problem. HCWs lack training on sign language, which necessitates training for HCWs through their training years and at the health care settings (Moore et al., 2007).

The findings revealed that HCWs struggle to communicate with hard of hearing patients who are South Africans, and especially those who are not from South Africa, because of the various languages which create a barrier. Orrie and Motsohi (2018) state that South Africa is a multilingual society with one official language and that many South Africans do not speak English. They add that approximately $80 \%$ of health care consultations in South Africa are carried out across linguistic and cultural barriers, and the language barrier has become normative and even invisible. It compromises patient quality of care where those who cannot communicate with HCWs are less likely to adhere to treatment, to seek care or follow-up appointments or receive preventive services. HCWs rank language barriers between them and patients who speak other languages as a greater problem (Orrie and Motsohi, 2018). Sibiya (2018) concurs with the findings by stating that the difference in language is a widely acknowledged as a major barrier to effective communication and accessing health care. When the nurse and the patient do not share a common language, interaction between them is strained and very limited (Sibiya, 2018).

Participants reported having difficulty with various languages in the health care setting. General and specific communication skills are needed by health professionals who work in the health care industry, both with normal and hearing-impaired patients. Language is an important enabler of right to health. It allows access to health care information, both preventative and curative, and allows for health care to be provided with informed consent from the user. Language is essential to developing a therapeutic relationship with a patient, yet language is a barrier between deaf patients and HCWs and is one of the main challenges in accessing health care (Machado et al., 2015).

A study done by Dickson and Magovan (2014), shows that nurses often communicate inappropriately and lack the knowledge and skills to understand the communication with deaf people. When a nurse in a health institution meets a patient with hearing impairment, efficient, mutually clear and understandable communication becomes a 
problem. Some of the participants indicated not feeling comfortable communicating with hard of hearing patients due to the lack of knowledge on SASL (Dickson \& Magovan, 2014)). According to Lubjubicic et al. (2017), a feeling of discomfort and insecurity indicates a need for education about ways of communication with deaf people in order to reduce the difficulties and to develop better communication at mutual pleasure (Lubjubicic et al., 2017).

Communication is indicative of quality of life, therefore, when a health care professional knows how to communicate properly with deaf patients; they are promoting a humanized and focused assistance (Alselai \& Alrashed, 2015). The skills to deal with people who do not share oral language and culture in the world environment is not taught in schools. Professionals may not be prepared to meet the needs of these patients. The theoretical concept applied from the Peplau's interpersonal relations theory is the identification of health problem, which has been stated above by the participants, which is the language barrier that they experience with various patients who communicate in sign language in difference languages (Alselai \& Alrashed, 2015).

Participants, in the current study, stated that they preferred using family members to interpret for the patient as it easy to communicate with the hard of hearing patients and less time consuming. Participants indicated having the hard of hearing patient to come with a family member to assist in communication. Using family members as interpreters may impede both effective and physician/patient confidentially care (Machado et al., 2015). The results of the study revealed that it is time consuming when the HCW communicates directly with hard of hearing patients, especially since only a few of them are trained on SASL. Other means of communication, like writing, also depend on whether the patient is literate. Due to lack of time, nurses sometimes avoid direct communication and communicate with a deaf person through an intermediary, most often a family member, although using family members can cause problems for patient confidentiality or in protecting the patient from dispiriting information care (Machado et al., 2015).

According to the study done by Ali (2012), most interpreters, such as friends and family members, are informal. Family members are support for the deaf and hard of hearing patients, helping the patients to navigate the health care system and being an interpreter. However, it also became apparent that the family members provide valuable help to the HCWs themselves. HCWs actively seek them out because their presence makes the HCWs feel more comfortable and satisfied with the encounter. While interpreters are helpful in overcoming language barriers, inappropriate use of family members as interpreters may rob a patient of his or her autonomy and the right to confidentiality (Ali, 2012).

The findings of the study that was conducted by Blake et al. (2014) revealed that deaf patients acquire health care knowledge from family members who may not have access to correct information either. This inaccurate information leaves the deaf patient with misunderstandings and misconceptions, which can have a long-term impact on their health (Blake et al., 2014). According to Benjamin (2016), in South Africa common language practices to address in health care include involving the use of family members and nurses as informal interpreters (Benjamin 2016). The theoretical concept used in these themes is the identification of a health problem which is involving family members during consultation for hard of hearing patients which affects the patient privacy and confidentiality. However, trained sign language interpreters are needed for such a service (Benjamin, 2016). Participants in the study reported having communication challenges with hard of hearing patients and used gestures, writing and other HCWs to communicate [12]. Using pen and paper is the main method used when HCWs communicate with deaf or hard of hearing patients. HCWs' skills in communicating with deaf and hard of hearing patients require attention (Machado et al., 2015).

Participants reported using writing and gestures to communicate with deaf patients. A study by Hunter-Adams and Rother (2017) revealed that writing is one of the best ways to get a message across to a deaf person, especially when speech does not seem to be conveying the desired message [10]. Lack of professional medical interpretation affects adequate health care provision. This research shows that nurses communicate with deaf people mainly by gesturing and writing but cannot clearly assess if the deaf people have understood their message (Hunter-Adams and Rother, 2017).

Participants revealed that, sometimes, they have to raise their voices to pass a message to the hard of hearing patients. According to Hemsley et al. (2012), although speaking louder to a deaf person or hard of hearing person is a natural inclination, it rarely, if ever, enhances comprehension. A profoundly deaf person will still hear nothing, no matter how loudly a message is spoken. He adds that, patients who wear hearing aids may experience both distortion and pain when shouting or when abnormally loud speech is amplified (Hemsley et al., 2012). The theoretical concept applied in this theme was the identification of a health problem which were the communication challenges the HCWs have with hard of hearing patients.

Participants revealed the various measures that they use to maintain confidentiality, such as providing information 
about confidentiality and ensure that the environment is suitable for hard of hearing to maintain confidentiality during consultations. Ajaj (2012) concurs that hard of hearing patients should feel secure and protected when communicating with HCWs in safe and private conditions. Patient confidentiality should be carefully maintained, and this can be achieved by restricting the relationship between HCWs and the patient to the medical issue.

During consultation, a conversation between the HCW and hard of hearing or deaf patient, via a sign language interpreter is confidential. When a family member is part of communicating the treatment plan to the hard of hearing patient, he or she is viewed as part of the health care team. They are advised to keep all medical information confidential. Patient information is very sensitive, so the prevention of disclosure and preservation of personal information leads to development of trust and confidence between HCWs and hard of hearing patients and strengthens the relationship. In this theme. the theoretical concept applied is the patient's sense of belonging which needs to be achieved by HCWs through maintaining confidentiality (Ajaj, 2012).

Study findings study showed that HCWs preserve the HCW-patient relationship through various forms of communication, including non-verbal communication such as maintaining eye contact (Machado et al., 2015). HCWs who use facial expressions, gestures and animation to supplement spoken messages are generally more readily understood than those who do not. Facial expressions are also crucial in conveying the intensity of a message. Hand gestures, signing and demonstrations are frequently used with most of the HCWs, showing enthusiasm for sign language. They further state that this a valuable skill as deaf patients recognise the use of basic signing as a genuine attempt by the HCWs to achieve good communication, furthering the development of a therapeutic bond (Blake, 2014).

Trust between HCWs and the patients is usually the pioneer of a strong relationship in which mutual respect emerges, which protects patient's medical confidentiality (Ajaj, 2012). HCWs adopted many strategies to encourage better communication. They speak slowly and clearly, maintain eye contact, raise their voices when appropriate and speak to the better hearing ear. The theoretical concept from the Peplau's interpersonal relations theoretical framework used in this theme is the nurse-patient relationship. In this case, it includes the relationship between HCWs and hard of hearing patients which ought to be maintained despite the communication challenges the participants' experience (Orrie \& Motsohi, 2018).

\section{Conclusions and Further Research}

The aim of the study was to explore and describe the experiences of HCWs regarding maintenance of confidentiality concerning hearing challenged people in selected public health settings in eThekwini District, KZN in South Africa. This study is another contribution to address issues related to the maintenance of confidentiality for hard of hearing patients. This study does not claim to exhaust the subject but to raise attention to new investigations in other realities. More research in the larger representative population of HCWs and hard of hearing patients is necessary to make the health care system more accessible and communication with HCWs more satisfying and effective. A doctoral study is necessary that will develop a practice framework to facilitate communication between hard of hearing patients and HCWs. A practice framework needs to be developed to promote communication between hard of hearing patients and HCWs.

\subsection{Recommendations}

The Department of Health, in collaboration with Department of Higher Education and Training, should consider introducing basic sign language studies in the curriculum for basic, secondary level, and tertiary level so that graduates at each level will be equipped with basic sign language communication. In order to enhance the use of sign language, policy should be directed and enforced for all HCWs to acquire the necessary education on basic sign language within the working environment and during their training school sessions. Public hospitals should employ qualified sign language interpreters in each health facility to assist during consultations to avoid the involvement and use of family members as interpreters, which impedes the hard of hearing patient's confidentiality.

The government should provide more schools for the deaf so they can also express themselves well in the sign language and writing, reducing the need for family members to act as interpreters. The leaders of the deaf associations should promote and encourage the deaf to go to the health facilities with their own interpreters to assist the deaf on arrival and should make them aware of their presence and notify the HCWs that they are deaf. Sign language posters should be displayed in all consultation rooms with a few common and practical signs to show deaf people that an attempt is being made to communicate. Development of deaf-friendly health care-based technologies to assist the communication between HCWs and deaf patients. 


\section{Competing Interests Statement}

The authors declare that they have no competing interests.

\section{References}

Ajaj, S. (2012). Improving patient confidentiality systems in Libya using UK experience. D. Tech. University of Gloucestershire.

Ali, J. D. (2012). Providing interpreters for patients with hearing disabilities: ADA requirements. Innovative Clinical Neuroscience, 9(9), 30-33. Retrieved 18 August, 2019, from https://innovationscns.com

Alselai, S. A., \& Alshred, A. M. (2015). Patient-nurses-relationship with deaf and hard of hearing (D\&HH) population. IOSR Journal of Nursing and Health Science, 4(1), 81-85. https://doi.org/10.9790/1959-04128185

Anney, V. N. (2014). Ensuring the quality of findings of qualitative research: looking at trustworthiness criteria. Scholarlink Research Institute Journals, 5(2), 272-281. Retrieved 12 March, 2019, from https://pdfs.sematicscholar.org

Babbie, E., \& Mouton, J. (2001). The practice of social research. Cape Town: Oxford University Press.

Benjamin, E., Swartz, L., Hering, L., \& Chiliza, B. (2016). Language barriers in health lessons from the experiences of trained interpreters working in public sector hospitals in Western Cape. South African Health Review, (1), 73-81. Retrieved 4 April, 2019, from https://www.hst.org.za

Blake, E., Glaser, M., \& William, D. T. (2014). Toward communication and information access for deaf people. Neuropsychological Rehabilitation, 54, 9-11. https://doi.org/10.18489/sacj.v54i0.236

Creswell, J. W. (2009). Research design: qualitative, quantitative and mixed approaches ( ${ }^{\text {rd }}$ ed.). Los Angeles: SAGE publications.

Dickson, M., \& Magowan, R. (2014). Meeting deaf patients' communication needs. Nursing Times, 110(49), 12-15. $\begin{array}{lllll}\text { Retrieved } & 18 & \text { August, } & \text { 2019, }\end{array}$ https://www.nursingtimes.net/clinical-archive/long-term-conditions/meeting-deaf-patientscommunication-n eeds-28-11-2014/

Grove, S. K., Burns, N., \& Gray, J. R. (2014). Understanding nursing research: Building evidence-based practice $\left(6^{\text {th }}\right.$ ed.). St Louis. Elsevier Saunders.

Hemsley, B., Balandin, S., \& Worrall, L. (2012). Nursing the patient with complex communication needs: time as a barrier and a facilitator to successful communication in hospital. Journal of Advanced Nursing, 68, 116-126. https://doi.org/10.1111/j.1365-2648.2011.05722.x.Epub.b

Hunter-Adams, J., \& Rother, H. (2017). A qualitative study of language barriers between South African health care providers and cross-border migrants. BMC Health Services Research, 17(97), 1-9. https://doi.org/10.1186/s12913-017-2042-5 (Accessed 11 July 2019).

Lincoln, Y. S., \& Guba, E. G. (2000). Competing paradigms in qualitative research. Thousand Oaks, California: Sage.

Lubjubicic, M., Zubcic, S., \& Sare, S. (2017). Communication between nurses and deaf people in health institutions. Research Rate, 5(1052), 1-8. https://doi.org/10.12955/cbup.v5.1052

Machado, W. C. A., Shubert, C. O., de Aguiar Prado, A. R., Fernades, E. M., Tonini, T., \& Leoni, M. G. (2015). The teaching of sign language in undergraduate nursing courses: A descriptive study as an attribute to the integrity of care. Scientific Research Publishing, 7, 1824-1832. https://doi.org/10.4236/health.2015.714200

McClelland, R., \& Thomas, V. (2002). Confidentiality and security of clinical information in mental health practice. Advances in Psychiatric Treatment, 8, 291-296. https://doi.org/10.1192/apt.8.4.291

Moore, I. N., Snyder, S. L., \& Qi An, A. (2007). Confidentiality and privacy in health care from patient's perspective: does HIPPA help. Health Matrix: The Journal of Law Medicine, 179(2), 214-272. Retrieved 16 May, 2019, from https://www.researchgate.net/publication/5526665_Confidentiality_and_privacy_in_health_care_from_the_ patient's_perspective_does_HIPAA_help

O'Brien, J., \& Chantler, C. (2003). Confidentiality and the duties of care. Journal of Medical Ethics, 29, 36-40.

Penn, C., \& de Andrade, V. (2017). Informed consent and deafness in South Africa: guidelines for clinicians and 
researchers. South African Journal of Bioethics Law, 10(2), 58-61. https://doi.org/10.1136/jme.29.1.36.

Richardson, K. J. (2014). Deaf culture: competencies and best practices. The Nurse Practitioner, 39(5), $20-28$. https://doi.org/10.1097/01.NPR.0000445956

Sibiya, M. N. (2018). Nursing. Effective communication in nursing. Nursing, Nilgun Ulutasdemir, IntechOpen, https://doi.org/10.5772/intechopen.74995

Simasiku, L., \& Nghitanwa, E. (2017). Experiences of registered nurses on communication with Deaf patients at Katutura State Hospital, Namibia. International Journal of Medicine, 5(1), 82-86. https://doi.org/10.14419/ijm.v5i1.7301

South Africa. Department of Health. (2003). The National Health Act 61 of 2003. Pretoria: Department of Health.

\section{Copyrights}

Copyright for this article is retained by the author(s), with first publication rights granted to the journal.

This is an open-access article distributed under the terms and conditions of the Creative Commons Attribution license (http://creativecommons.org/licenses/by/4.0/). 